Editorial

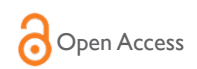

CrossMark

\title{
Eustachian tube catheterization
}

Volume 3 Issue 2 - 2015

\section{Introduction}

Although Eustachian tube obstruction (ETO) as one of the principal causes of 'hearing loss', and/or 'ear fullness', and/or 'tinnitus', and/ or 'headache (including otalgia)', and/or 'vertigo', has already been recognized by many well-respected senior doctors for a long time, it has still received only scant attention both in the literature and in practice. $^{1,2}$

Pressure differences between the middle ear and the atmosphere cause temporary conductive hearing loss by decreased motion of the tympanic membrane and ossicles of the ear. ${ }^{3}$ This point includes clue for explaining the mechanism of tinnitus due to Eustachian tube obstruction. ${ }^{1}$ Improvement of tinnitus after Eustachian tube catheterization, can mean that the tinnitus is from the hypersensitivity of cochlear nucleus following decrease of afferent nerve stimuli owing to air-bone gap. ${ }^{1,4}$

The middle ear is very much like a specialized paranasal sinus, called the tympanic cavity; it, like the paranasal sinuses, is a hollow mucosa-lined cavity in the skull that is ventilated through the nose. ${ }^{5}$ Tympanic cavity and mastoid cavity are named on the basis of anatomy. However, if we view things from a different angle, we can regard them as one of 'paranasal sinuses', called 'tympanic sinus' like maxillary or frontal or ethmoid or sphenoid sinus in a view of physiology and function. ${ }^{1}$ This point is the reason why I support the theory of 'sinus headache' when I need to explain the cause of headache from Eustachian tube obstruction. ${ }^{1}$ The bony labyrinth, or osseous labyrinth, is the network of passages with bony walls lined with periosteum. The membranous labyrinth runs inside of the bony labyrinth. There is a layer of perilymph fluid between them. The three parts of the bony labyrinth are the vestibule of the ear, the semicircular canals, and the cochlea. ${ }^{6}$

In the middle ear, the energy of pressure waves is translated into mechanical vibrations by the three auditory ossicles. Pressure waves move the tympanic membrane which in turns moves the malleus, the first bone of the middle ear. The malleus articulates to incus which connects to the stapes. The footplate of the stapes connects to the oval window, the beginning of the inner ear. When the stapes presses on the oval window, it causes the perilymph, the liquid of the inner ear to move. ${ }^{6}$ The vestibular system of the inner ear is responsible for the sensations of balance and motion. It uses the same kinds of fluids and detection cells (hair cells) as the cochlea uses, and sends information to the brain about the attitude, rotation, and linear motion of the head. The type of motion or attitude detected by a hair cell depends on its associated mechanical structures, such as the curved tube of a semicircular canal or the calcium carbonate crystals (or otolith) of the saccule and utricle.

Vertigo from ETO can be explained by an increase of inner pressure of the vestibular organ. It is because negative middle ear pressure gives rise to the retraction of the tympanic membrane, and it makes the stapes push against the oval window. ${ }^{1}$ It seems obvious that obstruction of the Eustachian tube somehow disturbs the air pressure and causes stimulation of the perilymph, which interferes with normal balance as maintained by the labyrinthine mechanism. ${ }^{7}$ There are many other conditions which may cause vertigo, but since Eustachian tube obstruction is one of the most obvious, and also the most easily corrected, every patient with symptoms of vertigo, and/or nausea, and/or vomiting, and/or perspiration should be subjected to the therapeutic test of inflation of the tubes as a first step in a thorough clinical investigation. ${ }^{2,7}$

Some researchers mention that an example of dizziness induced by middle ear pressure fluctuation is 'alternobaric vertigo' -- such as occurs in people who can 'clear' one ear, but not the other. ${ }^{8-12}$ This is mainly a problem in scuba divers and airplane pilots. It seems likely that this syndrome due to ETO is either caused by asymmetry in inner ear pressure accompanying changes in middle ear pressure, or due to displacement of otolithic membranes associated with displacement of the ossicular chain accompanying an inequity between middle ear and external ear pressure. They think that the pressure sensitivity is a symptom, not a disease. So it tends to be regarded as a normal variant. ${ }^{8-12}$

However, we need to study a question in its broader aspects. Especially in cases complaining of nausea, vomiting, and perspiration accompanied concomitantly by vertigo, which are attributable to ETO, their physicians tend to fail to recognize the cause in the emergency room or outpatient clinic., ${ }^{2,7}$ While vertigo originated from ETO is a distinctive clinical entity, doctors ignore the possibility that the violent symptoms of dizziness, nausea, vomiting and perspiration can be originated from ETO. ${ }^{2,7}$ The reason is that some of the physicians, audiologists, otolaryngologists or even otologists, are likely to think in terms of disturbances in the digestive, circulatory, nervous systems or only vestibular organ function, and usually examine them without considering other possibilities ${ }^{2,7}$. What's even worse, many special clinical tests of vestibular function are performed even in that case such as vertigo due to ETO. ${ }^{2}$ Here, we need to remember this clear proposition that ETO is the obvious cause for 'loss of vestibular function'. ${ }^{2}$

Recognition of the head's movement relative to the body is provided by the linear (otolithic macula) and angular (semicircular 
canals) acceleration receptors of the inner ear. Electrical activity generated within the inner ear travels along the vestibular nerve (primary afferent neuronal pathway) to the central vestibular nuclei of the brainstem, forming second-order neuronal pathways that become the vestibulo-ocular reflex (VOR), the vestibulospinal tracts, and the vestibulocerebellar tracts. Pathways derived from vestibular information also travel to the brainstem emetic centers (or solitary nucleus), which serves to explain vegetative symptoms such as nausea, vomiting, and perspiration that a patient typically experiences following an "Acute Unilateral Vestibular Loss " originating from "Acute Unilateral Eustachian Obstruction".,13 We can realize that electrical activity generated by ETO, travels to the brainstem emetic centers, and cause vegetative symptoms such as nausea, vomiting, and perspiration after all. ${ }^{2}$

With a loss of unilateral vestibular function, the patient acutely experiences the sensation of true vertigo from interruptions of VOR pathways and tends to lie perfectly still, as any movement aggravates vegetative symptoms such as nausea and vomiting that arise from the emetic centers. ${ }^{13}$ Nystagmus beating away from the side of lesion is the cardinal physical sign that obeys Alexander's law. ${ }^{14}$ Interruption in vestibulospinal tract pathways causes the patient to fall or list toward the affected side. Findings of ipsilateral hemispheric cerebellar dysfunction presenting with behaviors such as pastpointing, an inability to perform rapid alternating movements (dysdiadochokinesis), and gait ataxia reflect acute vestibulo cerebellar tract involvement. ${ }^{13}$ The vertigo is caused in most instances and perhaps in all, by unilateral Eustachian obstruction or by more complete obstruction on one side than the other. ${ }^{7}$ The direction of the gait furnishes a clue to the side which may be affected, for in most of the cases the staggering is in the direction of the obstructed side. ${ }^{7}$ With compensation (implying the existence of a normal functioning CNS and contralateral peripheral vestibular system) there may be minimal symptomatology that is only brought out by very rapid head movements. ${ }^{13}$ The spontaneous nystagmus disappears, vegetative symptoms resolve, gait improves, and in the case of a chronic condition the patient may experience only a slight imbalance when turning quickly. ${ }^{13}$ This point can be the reason why any vestibular function test should never be performed before correction of ETO and without normalization of middle ear pressure and tympanic membrane retraction. ${ }^{2}$

Vertigo is not a feature of a bilateral vestibular loss even when it occurs in an acute fashion..$^{13}$ In an acute case, it doesn't matter if the obstruction of the Eustachian tube is unilateral or bilateral. Injury to the end organs as might occur in systemic amino glycoside vestibulo toxicity causes a bilateral loss of function that tends to be electrically symmetric at the level of the vestibular nuclei in the brainstem. Instead the patient tends to complain of oscillopsia (visual blurring with head movement) and imbalance. ${ }^{13}$ The gait is typically broad-based and ataxic, especially with eyes closed. Falls are not infrequent and in many instances the patient requires assistive devices for ambulation or is relegated to a wheelchair. Compensation is generally unlikely to occur despite the best efforts of vestibular rehabilitation therapy and a greater reliance on information from visual and proprioceptive receptors. ${ }^{13}$

In order to explain the mechanism of vertigo originated from Eustachian tube obstruction, it can be summarized in regular sequence as follows: Changes in middle ear pressure, negative middle ear pressure, an inequity between middle ear and external ear pressure, retraction of tympanic membrane, displacement of the ossicular chain, the stapes pushing against the oval window, stimulation of the perilymph, increase of inner pressure of vestibular organ, displacement of otolithic membranes, asymmetry in inner ear pressure, loss of unilateral vestibular function, interruptions of vestibulo-ocular reflex(VOR) pathways, and interference with normal balance as maintained by the labyrinthine mechanism., ${ }^{1,-13}$ Now, it is reasonable to ask some questions. What are the normal parameters of middle ear cavity pressure that does not have an impact on vestibular function ${ }^{2}$ ? What is the normal criterion for difference of pressure between both middle ear cavities that does not have an impact on vestibular function? So what is the reference point in order to differentiate between unilateral ETO and bilateral ETO?

When the onset of the symptoms of nausea and vertigo is sudden and severe, the diagnosis is more easily established, and, if the proper treatment is instituted promptly, the relief is striking and dramatic. ${ }^{7}$ The cases of insidious onset are the ones most likely to be overlooked. In these instances, because the gastrointestinal symptoms are predominant, the patients are likely to be subjected to various types of treatment over long periods for diseases of the digestive system 7. The gastrointestinal symptoms of the cases of insidious onset can also be regarded as symptoms of Laryngopharyngeal Reflux (LPR) or Gastroesophageal Reflux Disease (GERD). ${ }^{2}$ Hence, we can accept an Cartesian intuition that every variable symptom and disease associated with LPR or GERD should be included in the multiple symptoms and diseases originated from ETO. ${ }^{2}$ We also must pay attention to the necessity that the cases of insidious onset are first subjected to Eustachian tube catheterization. ${ }^{2}$

The symptoms of LPR are protean; however, the most common are hoarseness, globus pharyngeus, dysphagia, cough, chronic throat clearing, and sore throat. The most common manifestation of LPR is reflux laryngitis with or without granulation or granuloma formation. In addition, reflux has been reported to be associated with subglottic stenosis, laryngeal carcinoma, polypoid degeneration, laryngospasm, paradoxical vocal fold movement, and vocal nodules. Other manifestations in the head and neck that have been reported include asthma, sinusitis, and otitis media. ${ }^{15} \mathrm{I}$ believe that more variable symptoms related to both of LPR or GERD, e.g., back pain, diaphragmatic pain and numbness of fingers or toes, than many symptoms gaining academic recognition at present, need to be proved definitely.$^{2}$ In the patients having both LPR and ETO, ETO is not just a cause of LPR as seen above. On the contrary to this, LPR can be a cause of intrinsic mechanical obstruction of Eustachian tube. Recently several studies have shown an association between gastroesophageal reflux and Otitis Media with Effusion (OME), and a conclusion that LPR plays an important role in the pathogenesis of OME as gastric reflux reaches the middle ear through the nasopharynx and eustachian tube to cause OME. ${ }^{16}$ It seems obvious that reflux contents from stomach can reach the nasopharynx and the Eustachian tubes through esophagus, and block the tubes directly or cause inflammation of the mucous membrane lining of the Eustachian tube, or adhesion and its collapse. $^{2}$

Therefore, the concept of reciprocal causal relationship both of LPR and ETO, which cause a vicious cycle, is established distinctively. ${ }^{2}$ Treating patients regardless in an emergency room or outpatient clinic, a wide and diverse variety of symptoms originating from LPR or GERD and ETO, has to be considered with the mutual connection observantly . ${ }^{2}$ ETO should be subjected to the therapeutic test of inflation of the tubes as a first step in a thorough clinical investigation, ${ }^{1,7}$ because Eustachian tube catheterization may be of both diagnostic and therapeutic value. ${ }^{1}$ Moreover, every clinical test being used in the assessment of vestibular function should be performed in the state of 'ideally normal middle ear cavity pressure with perfectly equal balance between both ears' ${ }^{1,2}$ Especially in the 
caloric test which is based on the convection theory, this middle ear cavity air pressure is also the crucial variable which influences the temperature transmission to semicircular canal from external auditory meatus. This condition has to be checked before any other tests for any symptom and any disease accompanying LPR or GERD, and like loss of vestibular function, tinnitus, ear fullness, sensorineural hearing loss, headache, earaches, itching sensation of ear, migrainous vertigo, etc. ${ }^{1,2}$ With the above-mentioned necessity of Eustachian tube catheterization, I would like to introduce the method with devices like a 'Eustachian tube catheter' and a 'Toynbee diagnostic tube' in more detail, which is done in my clinic myself, although there are many ways of it. This procedure is more than a century old and was earlier routinely done to check patency of the Eustachian tube. ${ }^{1}$

It can be called as 'Rosenmuller method' ${ }^{17}$ The nasal tip is to be tilted upward with a finger of operator until the anterior nares are raised to the level of the floor of the nose. The tip of the catheter is inserted into the nose, then passed first upward and passed along the floor of the nasal cavity till it touches the posterior pharyngeal wall. The tip is now in the nasopharynx. ${ }^{1,17,18}$ At this point, the tip is rotated $90^{\circ}$ laterally so that it touches the Rosenmuller fossa behind the Eustachian tube in the lateral wall. The tip is pulled forward slightly, and passes over a gentle slope of the cartilaginous opening of the tube(or torus tubarius), and is rotated $45^{\circ}$ superior-laterally again so that it enters the opening of the Eustachian tube. ${ }^{1,17}$ A ventilator is attached to the other end of the catheter through a tube. ${ }^{1}$ Air pushed in pulsed mode from it can be heard rushing into the ear, if the Eustachian tube is patent. ${ }^{1}$ The operator can confirm the state and quality of this sound by auscultation with Toynbee diagnostic tube. The medial and lateral rotations that appear to complicate the procedure were actually designed to avoid the tubal elevation located behind the tubal opening since the tip cannot be seen once inserted into the nose. The original procedure was a blind one, with only the metal ring to indicate the direction of the catheter's tip. ${ }^{1}$ But the procedure can also be done under endoscopic guidance. ${ }^{1}$ Liquids and sprays are sometimes injected into the ear through the catheter; bougies are also passed into the tube in the same manner as the catheter or, if flexible bougies are used, they are passed through the catheter. As the tip of the bougie passes into the bony portion of the canal, the constriction of the isthmus can be felt $2.5 \mathrm{~cm}$ up from its mouth. The bougie should not be passed farther than $3 \mathrm{~cm}$ into the tube, otherwise, if the tympanum is entered, the ossicles are apt to be injured. ${ }^{18}$ The important thing to remember is that the method only tests patency of the Eustachian tube and does not reflect on its function. ${ }^{1}$

My medical experience over 20years has made me find out valuable opinions of the excellent researchers. As a clinician who inherited Eustachian tube catheterization through the apprenticeship, and should preserve it, I would like to present you this clear proposition at least as follows: With a view to 'ideally normal middle ear cavity pressure with perfectly equal balance between both ears', Eustachian tube catheterization may be of both diagnostic and therapeutic value., ${ }^{1,2}$ This procedure should be carried out only by an otolaryngologist who is skilled in the passing of Eustachian catheters and bougies. ${ }^{7}$ Eustachian tube catheterization is the most fundamental and essential technique for a veritable otolaryngologist.

\section{Acknowledgments}

None.

\section{Conflicts of interest}

Author declares there are nio conflicts of interest.

\section{Funding}

None.

\section{References}

1. Kim HY. Diagnosis \& Treatment of Mechanical Obstruction of Eustachian Tube. J Otolaryngol ENT Res. 2014; 1(1):00001.

2. Young Kim H. Reciprocal Causal Relationship between Laryngopharyngeal Reflux and Eustachian Tube Obstruction. $J$ Otolaryngol ENT Res. 2015;2(6):00046.

3. Rex S Haberman. Middle Ear and Mastoid Surgery. Thieme Medical $P u b$. New York, USA. 2004. p. 152.

4. Heller MF, Bergman M. Tinnitus aurium in normally hearing persons. Ann Otol Rhinol Laryngol. 1953;62(1):73-83.

5. Drake, Richard LV, Wayne T, et al.Illustrations by Richard; Richardson, Paul Gray's anatomy for students. Elsevier/Churchill Livingstone. Philadelphia, USA. 2005. p. 858.

6. Jan Schnupp, Israel Nelken and Andrew King. Auditory Neuroscience. MIT Press. USA. 2011.

7. Merica FW. Vertigo due to obstruction of the eustachian tubes. JAMA. 1942;118(15):1282-1284.

8. Klingmann C, M Knauth, Praetorius M, et al.Alternobaric vertigo-really a hazard? Otol Neurotol. 2006;27(8):1120-1125.

9. Subtil J, Varandas J, Galrão F, et al.Alternobaric vertigo: prevalence in Portuguese Air Force pilots. Acta Otolaryngol. 2007;127(8):843-846.

10. Tjernstrom O. Function of the eustachian tubes in divers with a history of alternobaric vertigo. Undersea Biomed Res. 1974;1(4):343-351.

11. Tjernstrom O. Further studies on alternobaric vertigo. Posture and passive equilibration of middle ear pressure. Acta Otolaryngol. $1974 ; 78(3-4): 221-231$.

12. Tjernstrom O. Middle ear mechanics and alternobaric vertigo. Acta Otolaryngol. 1974;78(5-6):376-384.

13. Roland PS, Rutka JA. Physiology of the vestibular system. In: Rutka JA (Ed.), Ototoxicity, BC Decker Inc., Hamilton, Ontario, Canada. 2004. p. $20-27$.

14. Doslak MJ, Dell'Osso L, Daroff RB. A model of Alexander's law of vestibular nystagmus. Biol Cybern. 1979;34(3):181-186.

15. Koufman JA, Aviv JE, Casiano RR, et al. Laryngopharyngeal reflux: Position statement of the Committee on Speech, Voice, and Swallowing Disorders of the American Academy of Otolaryngology- Head and Neck Surgery. Otolaryngol Head Neck Surg. 2002;127(1):32-35.

16. Al-Saab F, Manoukian JJ, Al-Sabah B, et al. Linking laryngopharyngeal reflux to otitis media with effusion: pepsinogen study of adenoid tissue and middle ear fluid. J Otolaryngol Head Neck Surg. 2008;37(4):565-571.

17. Noh K. Otolaryngology-Head and Neck Surgery, Problem and assessment. Ilchokak, Seoul, Republic of Korea. 1995. p.14-15.

18. Gwilym GD. Part 2. Pharynx. Introducing The Eustachian Catheter, Applied Anatomy: The Construction of The Human Body, J. B. Lippincott Company, Philadelphia, USA. 1913. 